\title{
Ultrafast nonlinear optics due to electron-hole plasma in bulk semiconductors
}

\author{
Hsin-Fei Meng ${ }^{\mathrm{a}, *}$, Chih-Ming Lai ${ }^{\mathrm{b}}$ \\ ${ }^{a}$ Institute of Physics, National Chiao Tung University, Hsinchu 300, Taiwan \\ ${ }^{\mathrm{b}}$ Department of Electrophysics, National Chiao Tung University, Hsinchu 300, Taiwan
}

Received 16 September 1996; received in revised form 10 March 1997; accepted 14 April 1997

\begin{abstract}
It is theoretically demonstrated that, in the presence of an electric potential landscape, the ground-state energy of the electron-hole plasma can be substantially lowered against the ground-state energy of the excitons. The electron-hole plasma is found to be unstable at lower carrier densities. The critical carrier density for the transition between these two states can be reduced. This reduction provides a new mechanism of nonlinear optics for bulk direct band-gap semiconductors, with picosecond relaxation time, large nonlinearity, and requiring low pump intensity.
\end{abstract}

PACS: 42.65.-k; 71.35.+z

Keywords: Electron-hole plasma; Semi conductors

The mechanisms for intensity-dependent refractive index have been one of the most important subjects for the researches on nonlinear optics. As the application on all-optical switching is concerned, not only the nonlinearity, but also the relaxation time, are of primary importance. For resonant excitations, whose nonlinearity is usually due to the absorption changes induced by the photo-excited quasi-particles, one faces a trade-off between the above two factors. The relaxation is limited by the lifetime of the excited quasiparticles. However, any mechanism that reduces the lifetime will frequently lower the concentration of the quasi-particles for a given excitation intensity, and therefore diminish the nonlinearity. On the other hand, enhancing the nonlinearity by increasing the lifetime will reduce the switching speed. In practice, the radiative lifetime of the quasi-particles usually sets the

* Corresponding author. ultimate limit for the switching time. In this paper, we present a new mechanism for resonant nonlinearity in bulk direct band-gap semiconductors under external electric potential. It does not suffer from the nonlinearity-relaxation time trade-off. This is because the relaxation time of this mechanism is not limited by the quasi-particle lifetime. Instead, it is only limited by the transition time between two different collective states, with distinct optical properties, of the quasi-particles. This transition time was found to be as small as a few picosecond. At the same time, the nonlinearity is enlarged.

First, take GaAs as an example. The nonlinear effect of bulk GaAs is appreciable only at very high excitation powers. For 3D materials, the principle mechanism for the exciton peak bleaching is the plasma screening effect. However, under resonant excitations, the electron-hole plasma (EHP) is formed only when the carrier density is as high 
as $10^{17} \mathrm{~cm}^{-3}[1,2]$, while the relaxation is limited by the nanosecond exciton lifetime [3]. For direct band-gap semiconductors, it is believed that the lifetime of the photo-excited carriers is too short to allow the formation of electron-hole liquid droplets. Instead, an uniform density is always maintained and continuously changed when the pump intensity is varied. This is true even when a plasma is formed [1]. Therefore, for a given electron-hole pair density, the ground state of the system is either an exciton molecule gas (EM), or an uniform EHP. At zero temperature, the system will stay in the state with lower energy, i.e., the ground state. The density dependence of ground state energy per pair of the EHP has been studied extensively $[4,5]$, while the energy of the EM is fixed except at very high densities. In particular, the energy is called the exciton Rydberg (Ry) for free excitons. As mentioned before, photoluminesence and absorption experiments lead to the conclusion that the EM persists up to densities around $10^{17} \mathrm{~cm}^{-3}$, beyond which the mean distance among the excitons becomes smaller than the exciton Bohr radius $a_{\mathrm{B}}$. Therefore the notion of excitons loses its meaning, and the EHP forms. This suggests that the energy of the EHP is never lower than that of the excitons. Clearly, any mechanism that reverses the relative energies of these two states, and thus stabilizes the EHP as the ground state at lower densities, will modify the nonlinear optical properties substantially.

We demonstrate that through the application of a slowly varying electric potential landscape that causes a long-range charge redistribution for the EHP and lowers its energy, the energy of the EHP can be lowered against the EM. The EHP then becomes the ground state for pair densities above a critical value $n_{1}$, which is intrinsic to the material and is as low as $10^{16} \mathrm{~cm}^{-3}$. Here $\mathrm{i}$ denotes instability (see below). When the density is even lower, the EHP becomes unstable and the system switches to EM. At such density, there is almost no phase-space filling (PSF) bleaching effect for the exciton absorption peak due to the presence of other excitons, while the plasma screening is still very effective, because it is still well above the Mott criterion $\left(5.4 \times 10^{14} \mathrm{~cm}^{-3}\right)[2,6]$. The consequence is that the relaxation time of the photo-induced absorption change is not limited by the lifetime of the carriers (still in the nanosecond range), but by the time the system takes to transform to its new ground state, as the density decays through the critical value $n_{\mathrm{i}}$. This time has been shown to be only a few picoseconds [7]. Besides GaAs, we will also discuss other semiconductors, e.g. $\mathrm{CuCl}$, for which the energy of the EHP does becomes lower than that of the EM for densities higher than a critical value $n_{\mathrm{c}}$, even without any external field.

Let us first briefly review the theoretical results on the ground-state energies of the EHP and EM without any electric field [5]. The ground-state energies per pair (in units of Ry), denoted by $\varepsilon$, of the EHP and EM as functions of the density are shown as the $e v_{0} /$ Ry $=0$ curve in Fig. 1. $v_{0}$, which measures the strength of the external potential, will be defined later. We use the dimensionless parameter $r_{\mathrm{s}}$ defined by $4 \pi\left(a_{\mathrm{B}} r_{\mathrm{s}}\right)^{3} / 3 \equiv 1 / n$, instead of the real electronhole pair density $n$. For the exchange-correlation energy of the EHP, we use the universal behavior of Vashishta and Kalia [5]. For the high-density correction of the EM curve, we use the results of a hard-core bose gas [8], with hard-core parameter chosen, quite phenomenologically and roughly, to be $20 \AA$. Our following discussions, however, do not depend on the detailed behavior of the $\varepsilon\left(r_{\mathrm{s}}\right)$ for the EM. A shift of $\Delta$ below the Rydberg for the EM ground-state energy is due to the binding energy of biexciton, and bound exciton. These curves are consistent with the experiments that the EHP forms only when the density is higher than $10^{17} \mathrm{~cm}^{-3}\left(r_{\mathrm{s}}<1\right)$. For $\mathrm{CuCl}$, the theoretical results [9], as shown in Fig. 2 (ev $/$ Ry $=0$ curve), are quite different. In particular, the EHP and EM curves cross each other, which is also consistent with the experiments [7].

To calculate the energy of the EHP under external potential, we use the density functional theory (DFT). As mentioned before, an uniform EHP is stable against small density fluctuations only when the mean electron-hole pair density $n$ is above certain value $n_{\mathrm{i}}$. We first demonstrate it by a stability analysis, then calculate, for $n>n_{i}$, the effect of external potential on the ground-state energy to the lowest order.

According to DFT, the ground-state energy is the minimum of a density functional (DF) $E\left[n_{\mathrm{e}}(r), n_{\mathrm{h}}(r)\right]$, where $n_{\mathrm{e}}(r)$ and $n_{\mathrm{h}}(r)$ are the electron and hole densities, respectively. The DF is usually written in the form [5]

$E\left[n_{\mathrm{e}}(r), n_{\mathrm{h}}(r)\right]=E_{\mathrm{es}}+E_{\mathrm{k}}+E_{\mathrm{xc}}$, 


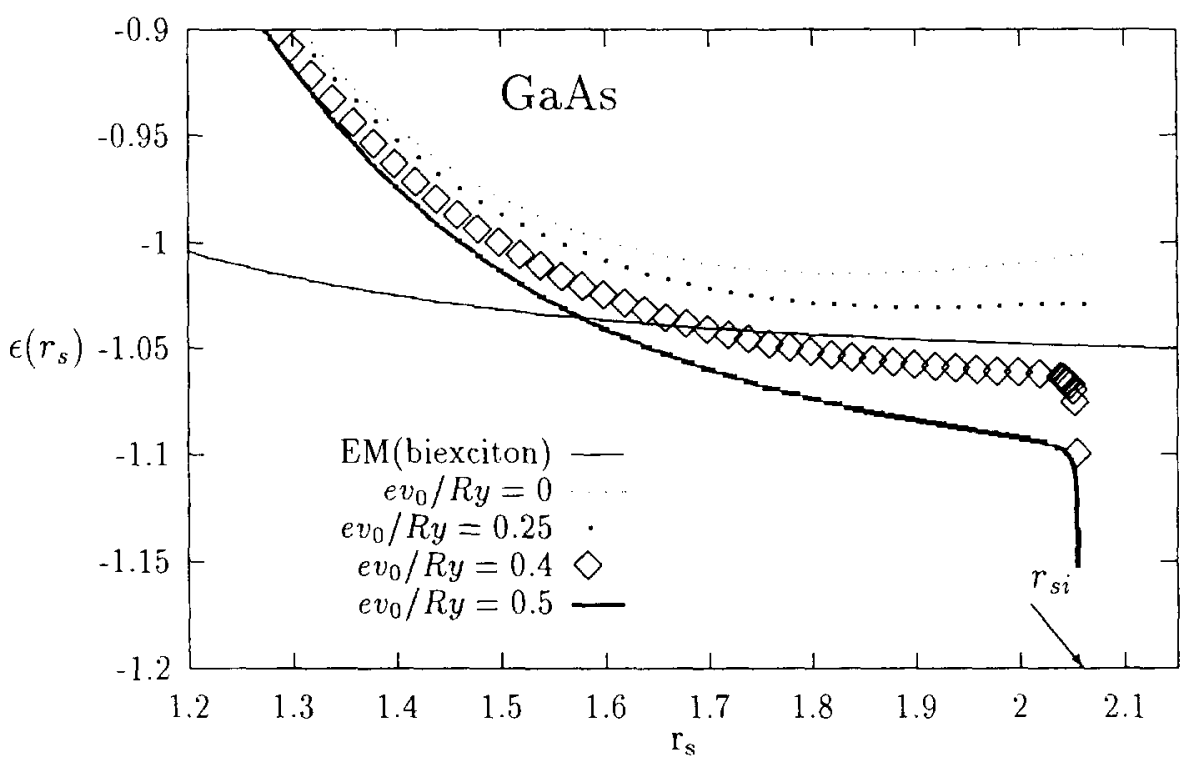

Fig. 1. The EHP ground-state energy $\varepsilon\left(r_{\mathrm{s}}\right)$ of GaAs for various $v_{0}$ 's are shown, together with the EM (biexciton) energy, which is independent of $v_{0}$. The sharp drop of $\varepsilon\left(r_{\mathrm{s}}\right)$ as $r_{\mathrm{s}}$ approaches $r_{\mathrm{si}}$ is due to the fact that one of the eigenvalues of $M$ approaches zero, and the linear response of the system to the external field diverges. One sees that as $e v_{0} / R y>0.4$, the ground state becomes the EHP for $r_{\mathrm{s}}<r_{\mathrm{si}}$, while the ground state remains the EM for $r_{\mathrm{s}}>r_{\mathrm{si}}$ for any $v_{0} . \Delta$ is taken to be $0.06 \mathrm{Ry}$ [3].

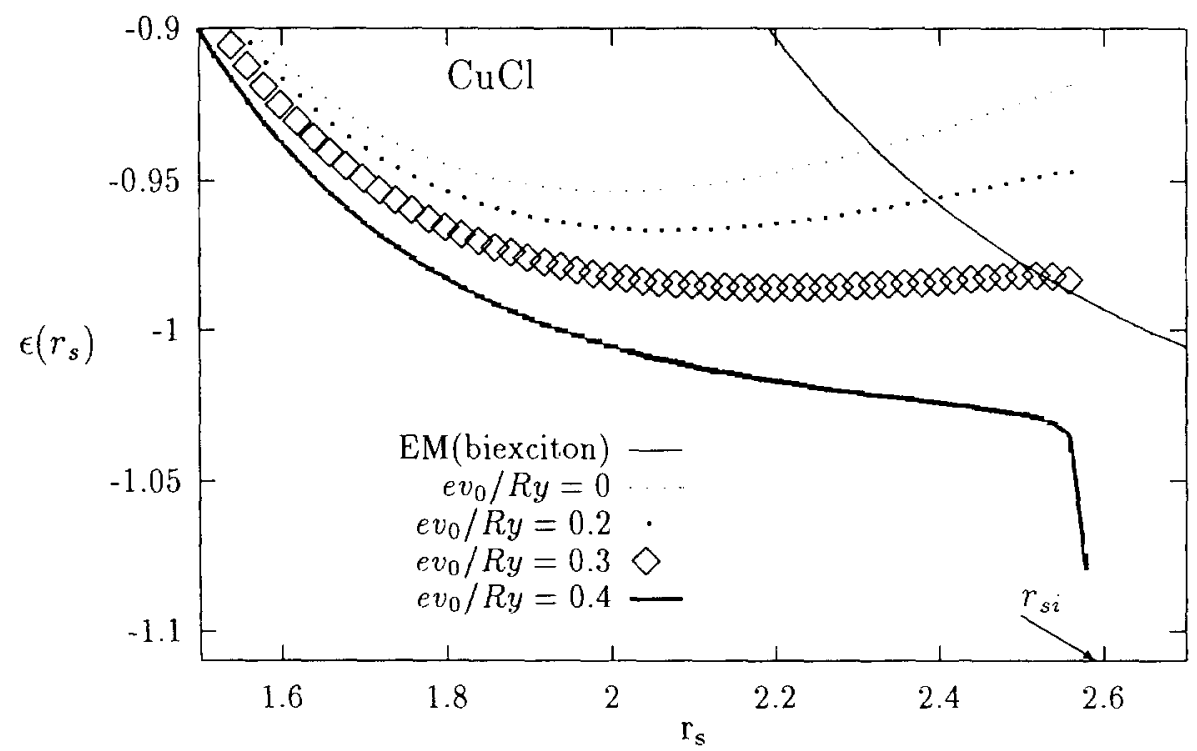

Fig. 2. Similarly to Fig. 1, the ground-state energy of the EHP for $\mathrm{CuCl}$ are shown for various $v_{0}$ 's. For $e v_{0} / \mathrm{Ry}<0.3$, the transition $r_{\mathrm{s}}$ between the EHP and EM increases with $e v_{0}$. For $e v_{0} /$ Ry $>0.3$, the transition happens at $r_{\mathrm{si}}(\simeq 2.59)$. See text for the associated nonlinear optical effects. $\Delta$ is taken to be $0.08 \mathrm{Ry}$ [11]. 
where the subscripts denote electrostatic, kinetic and exchange-correlation energies, respectively. The exact form of $E_{\mathrm{xc}}$ is unknown. However, for the purpose of stability analysis, we need to know it only up to the second order in the fluctuations of the electron and hole densities around their mean value $n$, i.e. $m_{\mathrm{e}}(r) \equiv n_{\mathrm{e}}(r)-n$ and $m_{\mathrm{h}}(r) \equiv n_{\mathrm{h}}(r)-n$. Assuming a local DF, we have [5]

$$
\begin{aligned}
E[n+ & \left.m_{\mathrm{e}}(r), n+m_{\mathrm{h}}(r)\right] \\
= & E[n, n]+\frac{1}{2} \int \frac{e^{2}}{\kappa\left|r-r^{\prime}\right|}\left[m_{\mathrm{h}}(r)-m_{\mathrm{e}}(r)\right] \\
& \times\left[m_{\mathrm{h}}\left(r^{\prime}\right)-m_{\mathrm{e}}\left(r^{\prime}\right)\right] \mathrm{d}^{3} r \mathrm{~d}^{3} r^{\prime} \\
& +\frac{1}{2} \int\left[\varepsilon_{\mathrm{e}}^{(2)} m_{\mathrm{e}}^{2}(r)+\varepsilon_{\mathrm{h}}^{(2)} m_{\mathrm{h}}^{2}(r)\right] \mathrm{d}^{3} r \\
& +\sum_{i=\mathrm{e}, \mathrm{h}} \Lambda_{\mathrm{i}}(n) \int\left|\nabla m_{\mathrm{i}}(r)\right|^{2} \mathrm{~d}^{3} r \\
& +\sum_{i, j=\mathrm{e}, \mathrm{h}} \Gamma_{i j}(n) \int \nabla m_{\mathrm{i}}(r) \cdot \nabla m_{j}(r) \mathrm{d}^{3} r \\
& +O\left(m_{\mathrm{e}}^{3}, m_{\mathrm{h}}^{3}\right) .
\end{aligned}
$$

Here $\kappa$ is the background dielectric constant and $e$ is the electron charge. $\varepsilon_{\mathrm{e}}$ and $\varepsilon_{\mathrm{h}}$ are the electron and hole parts of the kinetic and exchange-correlation energy per pair for a homogeneous system. The superscript "(2)" means the second derivative with respect to the densities, evaluated at $n$. The expressions for $\Lambda_{\mathrm{i}}(n)$ and $\Gamma_{i j}(n)$ can be found in Ref. [5]. For convenience, we express the DF in terms of the Fourier transforms of their density fluctuations $\tilde{m}_{\mathrm{e}}(k)$ and $\tilde{m}_{\mathrm{h}}(k)$. Substituting

$\tilde{m}_{\mathrm{e}, \mathrm{h}}(k)=\frac{1}{\sqrt{V}} \int \mathrm{e}^{-\mathrm{ik} \cdot r} m_{\mathrm{e}, \mathrm{h}}(r) \mathrm{d}^{3} r$,

with $V$ being the system volume, into Eq. (1), we have

$$
\begin{aligned}
E & {\left[n+m_{\mathrm{e}}(r), n+m_{\mathrm{h}}(r)\right] } \\
& =E[n, n]+\frac{1}{2} \sum_{k}\left(\tilde{m}_{\mathrm{e}}(k) \tilde{m}_{\mathrm{h}}(k)\right) M\left(\begin{array}{c}
\tilde{m}_{\mathrm{e}}(k) \\
\tilde{m}_{\mathrm{h}}(k)
\end{array}\right) .
\end{aligned}
$$

The linear terms vanish due to the constraints $\int m_{\mathrm{e}}(r) \mathrm{d}^{3} r=\int m_{\mathrm{h}}(r) \mathrm{d}^{3} r=0$. The two by two matrix $M$ has the form

$$
M=\left(\begin{array}{ll}
b & c \\
c & d
\end{array}\right),
$$

with the matrix elements

$b=\frac{4 \pi e^{2}}{\kappa k^{2}}+\varepsilon_{\mathrm{e}}^{(2)}+k^{2}\left(\Lambda_{\mathrm{e}}+\Gamma_{\mathrm{ee}}\right)$,

$c=-\frac{4 \pi e^{2}}{\kappa k^{2}}+k^{2} \Gamma_{\mathrm{he}}$,

$d=\frac{4 \pi e^{2}}{\kappa k^{2}}+\varepsilon_{\mathrm{h}}^{(2)}+k^{2}\left(\Lambda_{\mathrm{h}}+\Gamma_{\mathrm{hh}}\right)$.

The uniform electron and hole densities minimize the $D F$ if and only if both of the eigenvalues of $M$ are positive. It turns out that this is true only when $n$ is larger than a critical value $n_{\mathrm{i}}$, or when $r_{\mathrm{s}}$ is smaller the corresponding critical value $r_{\mathrm{si}}$. At the critical density, the determinant of $M$ is zero. The behaviors of the eigenvalues and the determinant, as functions of $r_{\mathrm{s}}$, are shown in Fig. 3. For $r_{\mathrm{s}}>r_{\mathrm{si}}$, the EHP is not stable. EM is expected to be the only possible state of the system.

Now we turn to the calculation of the ground-state energy of EHP under external potential $V(r)$, with the condition $r_{\mathrm{s}}<r_{\mathrm{si}}$, where both EHP and EM are possible. The new density functional $E_{V}$ can be easily obtained by adding a new external potential energy term $E_{\text {ex }}$ to the density functional $E$ of the uniform system. In terms of density fluctuations, we have

$$
E_{V}=E+E_{\mathrm{ex}}, \quad E_{\mathrm{ex}}=\sum_{k} e \tilde{V}(k)\left[\tilde{m}_{\mathrm{h}}(k)-\tilde{m}_{\mathrm{e}}(k)\right],
$$

where $\tilde{V}(k)$ is the Fourier transform of the external potential $V(r)$. The density configuration that minimizes $E_{V}$ can be obtained by the stationary conditions

$\frac{\delta E_{V}}{\delta \tilde{m}_{\mathrm{e}}}=\frac{\delta E_{V}}{\delta \tilde{m}_{\mathrm{h}}}=0$,

which leads to the matrix equation

$M\left(\begin{array}{c}\tilde{m}_{\mathrm{e}}(k) \\ \tilde{m}_{\mathrm{h}}(k)\end{array}\right)=\left(\begin{array}{c}-e \tilde{V}(k) \\ e \tilde{V}(k)\end{array}\right)$.

Assuming $V(r)$ is equal to $v_{0} \cos \left(k_{0} r\right)$, one can easily evaluate the ground-state energy per pair $\varepsilon$ to the order of $\left(v_{0}\right)^{2}$. The $r_{\mathrm{s}}$ dependances of $\varepsilon$ for several $v_{0}$ 's are plotted in Fig. 1. In our calculation, the wave length $2 \pi / k_{0}$ of the external potential is to be 100 times of the exciton Bohr radius. Such $V(r)$ can be achieved, in principle, by a sinusoidal doping modulation, with equal total number of donors and accepters. Other more practically feasible $V(r)$ should give qualitatively the same results, as long as a long-range charge 


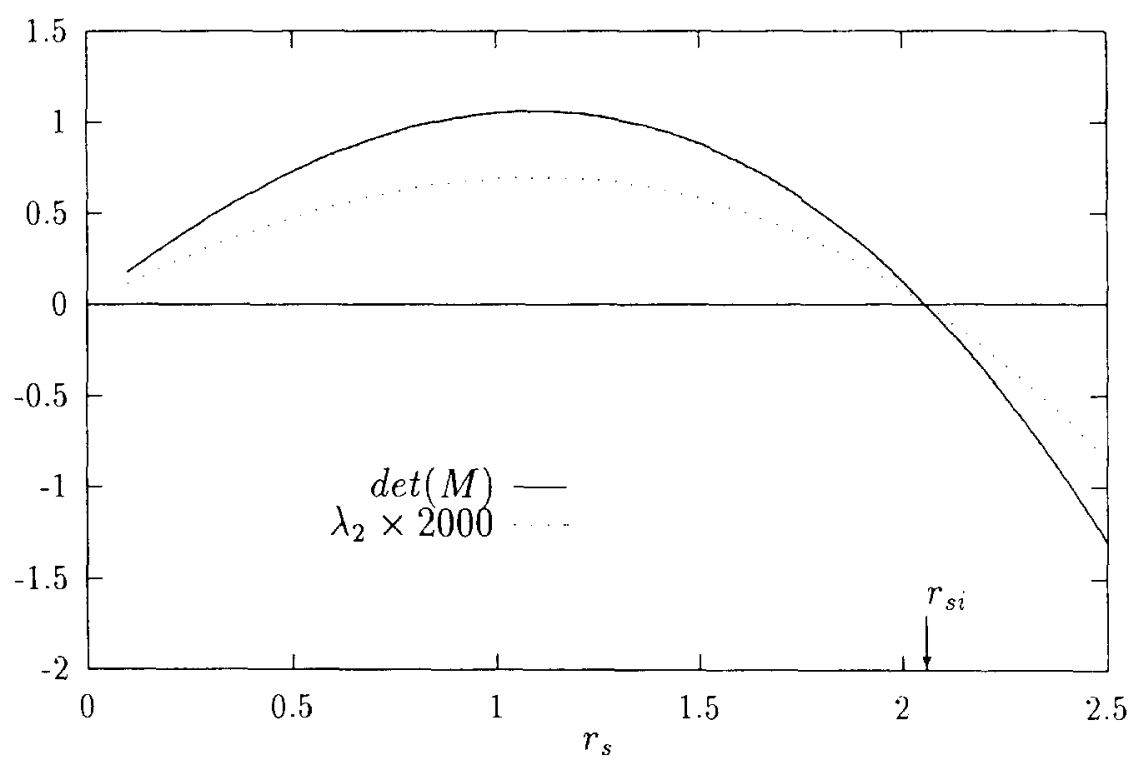

Fig. 3. The determinant $(\operatorname{det}(M))$, and one of the two eigenvalues $\left(\lambda_{2}\right)$, of the matrix $M$ are plotted as functions of $r_{\mathrm{s}}$. Another eigenvalue $\lambda_{1}$ stays basically a positive constant in this range of $r_{\mathrm{s}}$. When $r_{\mathrm{s}}>r_{\mathrm{si}}(\simeq 2.06), \lambda_{2}$ (and $\operatorname{det}(M)$ ) becomes negative, and the uniform EHP is not stable. The values of $\operatorname{det}(M)$ are in units of $\left(4 \pi a_{\mathrm{B}}^{3} \mathrm{Ry} / 3\right)\left(4 \pi e^{2} / \kappa k_{0}^{2}\right)$, and $\lambda_{2}$ in units of $4 \pi a_{\mathrm{B}}^{3} \mathrm{Ry}$.

redistribution is produced. In fact, our result is similar to the calculation for fast varying potentials [10]. The energy of the EM is assumed to be unaffected by the external field, because we are considering the longwavelength limit, where the EM's can be treated as point-like neutral particles. One of the most interesting features is that when the external potential is strong enough, the ground-state energy of the EHP can be substantially smaller than the EM energy for a wide range of $r_{\mathrm{s}}$ below $r_{\mathrm{si}}$. The divergency at $r_{\mathrm{si}}$ is due to the ignorance of all higher-order terms of the density fluctuations in Eq. (5). The reason is as follows. If we include the fourth-order terms of the density fluctuations $m_{\mathrm{e}}$ and $m_{\mathrm{h}}$ in Eq. (1) (no third-order terms due to inversion symmetry), there will be terms proportional to the third power of $\tilde{m}_{\mathrm{e}}$ and $\tilde{m}_{\mathrm{h}}$ with finite coefficients on the left-hand side of Eq. (5), in addition to the linear term. Therefore, even when the determinant of $M$ vanishes, the density fluctuations will not diverge. Instead, they will be proportional to the one-third of the external potential $V$. However, the inclusion of the higher-order terms does not change the order between the EHP and EM energies. It only removes the divergency near $r_{\mathrm{s}}=r_{\mathrm{si}}$ in Fig. 1. In fact, the linear response results are expected to be very accurate except at the immediate neighborhood of $r_{\mathrm{si}}$, because the relative density fluctuation is very small $(0.1 \%)$ for the external potentials considered here. Therefore, as long as nonlinear optics mechanism is concerned, linear response calculation is enough.

The anticipation of a mechanism for ultrafast nonlinear optics is now immediate. For GaAs, the critical value $r_{\mathrm{si}}$ separates two different states: EHP and EM. For $r_{\mathrm{s}}<r_{\mathrm{si}}$, the bleaching of the exciton absorption peak is due to the plasma screening of the Coulomb potential between the electrons and holes. On the other hand, for $r_{\mathrm{s}}>r_{\mathrm{si}}$, the bleaching is due to the PSF effect of the EM. Since $r_{\mathrm{si}}$ is much larger than one, while $n_{\mathrm{i}}$ is much above the Mott criterion, it is expected that the bleaching of the EHP is significantly more efficient than that of the EM [2]. In fact, under resonant pumping, when the carrier density is above the critical value $n_{\mathrm{i}}$, an EHP will be formed, and the exciton peak in the absorption spectrum will be completely bleached, while below $n_{\mathrm{i}}$, the exciton absorption peak remains basically unbleached (see Fig. 4). This nonlinear mechanism has an ultrafast decay time, which is equal to the time the EHP takes to cross the critical 


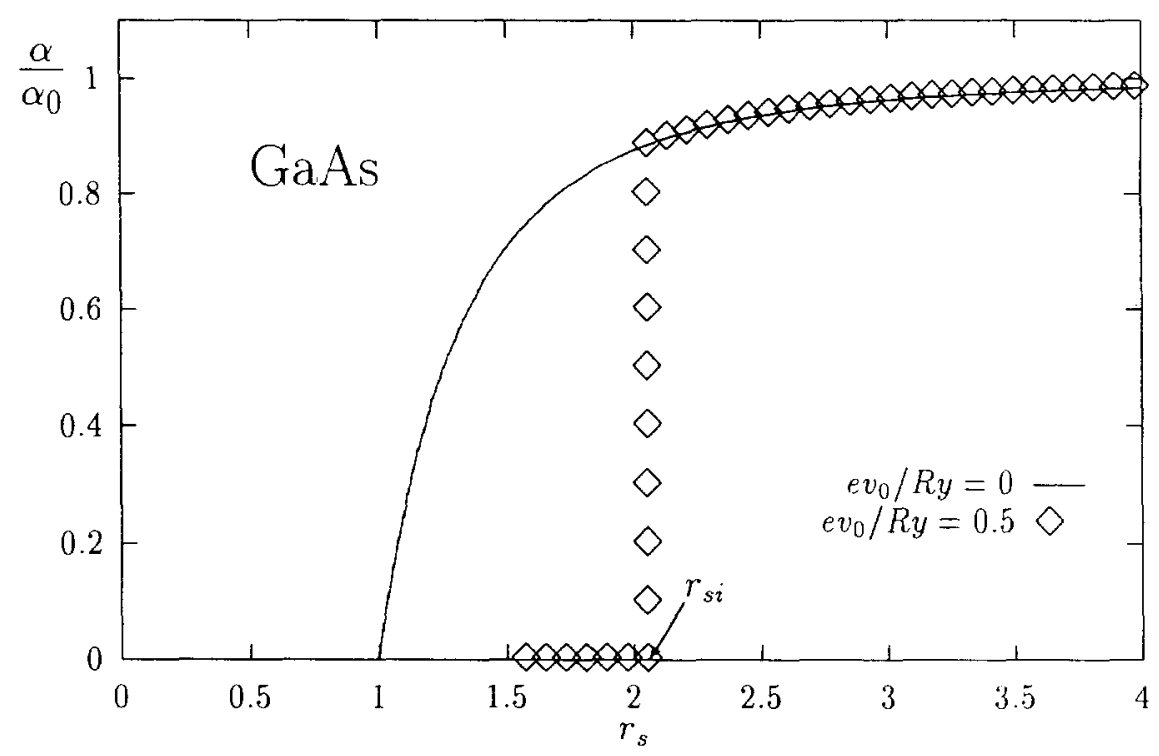

Fig. 4. The exciton bleaching factor $\alpha / \alpha_{0}$ are shown for two cases: $e v_{0} / \mathrm{Ry}=0$ and $e v_{0} / \mathrm{Ry}=0.5 . \alpha\left(r_{\mathrm{s}}\right)$ is the absorption coefficient at the $1 \mathrm{~s}$ exciton resonance when the pair density parameter is $r_{\mathrm{s}}$, and $\alpha_{0}$ is the absorption coefficient at the low excitation limit. For $e v_{0} / \mathrm{Ry}=0$, we have EHP when $r_{\mathrm{s}}<1$ and EM when $r_{\mathrm{s}}>1$. The gradual increase of $\alpha$ is due to the decrease of PSF effect. For $e v_{0} / \mathrm{Ry}=0.5$, we have EHP when $r_{\mathrm{s}}<r_{\mathrm{si}}$ and EM when $r_{\mathrm{s}}>r_{\mathrm{si}}$. The sharp increase of $\alpha$ is due to the sudden disappearance of the plasms screening effect. The bleaching of the EHP is assumed to be perfect, while the bleaching due to excitons is approximated as the $1-r_{\mathrm{s}}^{-3}$. Note that $r_{\mathrm{s}}^{-3}$ is the volume (and the phase space) fraction occupied by the existing excitons.

value $r_{\mathrm{si}}$, via radiative recombinations, to form $\mathrm{EM}$. It is found that, for uniform EHP, this time of the order of a few picoseconds [7]. In the presence of the external potentials considered here, the relative change in the carrier densities is only about $0.1 \%$ except at the immediate neighborhood of $r_{\mathrm{si}}$, as mentioned above. Therefore, the recombination process and the transition time are believed to remain the same as the uniform EHP. After the transition, a full exciton peak for the absorption will recover. Through the KramerKronig relation, a corresponding change of the refractive index will also occur.

For the case of $\mathrm{CuCl}$, there is a transition between EHP and EM happening at quite a high density ( $n \simeq 10^{20} \mathrm{~cm}^{-3}[7], r_{\mathrm{s}} \simeq 2.9$ ). The effect of the external potential is to reduce this transition density $n_{\mathrm{c}}$ (with $r_{\mathrm{c}}$ the corresponding dimensionless parameter) continuously, when $v_{0}$ is nonzero but small. When $v_{0}$ is further increased, the energy of the EHP for all $r_{\mathrm{s}}<r_{\mathrm{si}}$ becomes lower than the EM. $r_{\mathrm{si}}$ then takes over $r_{\mathrm{c}}$ and becomes the transition point between these two states, like the case of GaAs. Therefore, the con- trast of the exciton absorption peak between the two sides of the transition can be magnified, and the nonlinearity enhanced. Basic features are summarized in Fig. 2.

In conclusion, using linear response LDA calculations, we show that through the modulation of the ground-state energy of the EHP by an external potential, the EHP becomes the ground state of GaAs, except for low densities where an intrinsic instability occurs. For $\mathrm{CuCl}$, the critical density for the transition between EHP and EM can be reduced by the external potential. Based on the sharp contrast between the bleaching efficiencies of the EHP and low-density EM, a scheme for nonlinear optics, with picosecond decay time, is proposed. Because only the groundstate energy is discussed, in order to observe this effect, resonant pumping is required to produce cold excitons.

The authors are grateful for the support from the National Science Council of Taiwan under contract No. NSC85-2212-M-009-008. 


\section{References}

[1] T. Motiya, T. Kushida, Solid State Commun. 14 (1974) 245; See also H. Haug (Ed.), Optical Nonlinearities and Instabilities in Semiconductors, Academic, San Diego, 1988.

[2] G. Fehrenbach et al., Phys. Rev. Lett. 49 (1982) 1281

[3] G. 't Hooft et al., Phy. Rev. B 35 (1987) 8281.

[4] T. Rice, in: (Ed.), E. Ehenreich, Solid State Physics, vol. 32, Academic, New York, 1977.
[5] P. Vashishta et al., in: C. Jeffries, L. Keldysh (Eds.), ElectronHole Droplets in Semiconductors, Academic, New York, 1977.

[6] J. Shah, R. Leheny, W. Wiegmann, Phys. Rev. B 16 (1977) 1577.

[7] D. Julin et al., Appl. Phys. Lett. 45 (1984) 993.

[8] K. Huang, Statistical Mechanics, Wiley, New York, 1987.

[9] E. Hanamura, J. Lumin. 12/13 (1976) 119.

[10] Phys. Rev. B 39 (1989) 6264.

[11] D. Hulin et al., J. Lumin. 30 (1985) 290 and reference therein. 